\title{
Psychometric evaluation of the Adolescent Health Promotion Scale in Chile: differences by socioeconomic status and gender
}

Cristian A. Rojas-Barahona ${ }^{1}$, Jorge Gaete ${ }^{2}$, Esterbina Olivares ${ }^{3}$, Carla E. Förster ${ }^{1}$, Eugenio Chandia $^{4}$, Mei-Yen Chen ${ }^{5}$

${ }^{1}$ Cristian A. Rojas-Barahona, PhD.

Associate Professor

Faculty of Education, Pontificia Universidad Católica de Chile.

Vicuña Mackenna 4860, Macul.

Santiago, Chile.

Phone Number: +56 (2) 223545362

Email: crojash@uc.cl

${ }^{2}$ Jorge Gaete (Correspondent Author), MD, MSc, PhD.

Associate Professor

Faculty of Medicine, Universidad de los Andes.

Monseñor Álvaro del Portillo 12455.

Las Condes. Santiago, Chile.

Phone Number: +56 (2) 226181000

Email: jgaete@uandes.cl

Postdoctoral Research Fellow 
Centre for Global Mental Health

Department of Population Health

London School of Hygiene \& Tropical Medicine

Keppel Street

London, WC1E 7HT

Phone Number: +44 (0) 2076368636

Email: jorge.gaete@1shtm.ac.uk

${ }^{3}$ Esterbina Olivares, MSc, RN.

Adjunct Professor

School of Nursing (Campus San Felipe), Universidad de Valparaíso.

Camino La Troya with El Convento

San Felipe, Chile.

Phone Number: +56 (34) 2431221

Email: eolivaresv@yahoo.com

${ }^{4}$ Carla E. Förster, PhD.

Associate Professor

Faculty of Education, Pontificia Universidad Católica de Chile.

Vicuña Mackenna 4860, Macul.

Santiago, Chile.

Phone Number: +56 (2) 223545326

Email: ceforste@uc.cl 
${ }^{4}$ Eugenio Chandia, MSc.

Instructor Professor

Faculty of Maths, Pontificia Universidad Católica de Chile.

Vicuña Mackenna 4860, Macul.

Santiago, Chile.

Phone Number: +56 (2) 223544511

Email: echandia@uc.cl

${ }^{5}$ Mei-Yen Chen, $\mathrm{PhD}$, RN.

Professor, Dean, College of Nursing

Chang Gung University of Science and Technology

261, Wen Hwa 1st Rd, Kwei-Shan,

Tao-Yuan, 33303 Taiwan R.O.C.

Phone Number: 886-3- 211-8999 \#3111

Email: meiyen@gw.cgust.edu.tw

Conflicts of Interest and Source of Funding: Authors have no conflict of interest to declare. 